\title{
Epstein-Barr virus, the immune system, and associated diseases
}

\author{
Mei-Ru Chen* \\ Epstein-Barr Virus Group, Graduate Institute and Department of Microbiology, National Taiwan University, Taipei, Taiwan
}

\author{
Edited by: \\ Joseph K. Li, Utah State University, \\ USA \\ Reviewed by: \\ Dale L. Barnard, Utah State University, \\ USA \\ Joseph K. Li, Utah State University, \\ USA \\ *Correspondence: \\ Mei-Ru Chen, Graduate Institute and \\ Department of Microbiology, College \\ of Medicine, National Taiwan \\ University, No. 1, First Section, Jen-Ai \\ Road, Taipei 100, Taiwan. \\ e-mail:mrc@ntu.edu.tw
}

Host immune system is designed (or evolved) to fight against different pathogens. Many viruses infect the immune cells for the propagation of new progenies, thus the infection may modulate the host immune homeostasis. It has been more than 45 years since the discovery of Epstein-Barr virus (EBV) from a Burkitt's lymphoma derived cell line. The ability of EBV to transform primary B cells in vitro leads to the suggestion for its oncogenic potential. However, except the clear understanding of the role of EBV in post-transplantation lymphoproliferative disease, it remains ambiguous why such a ubiquitous virus causes malignant diseases only in a very small subset of individuals. Possible explanation is that EBV may cooperate with other environmental and host genetic factors and lead to the development of EBV associated neoplastic diseases. In addition to infecting B cells, recent studies revealed that EBV may impact host immune system more broadly than previously thought, for example the development of regulatory NKT subsets. Instead of an intensive review, this article aims to provide a linkage to recent advances on the interplay between EBV and host immune system and to inspire further studies on EBV related diseases, especially autoimmune diseases.

Keywords: Epstein-Barr virus, immune system, autoimmune disease

\section{EPSTEIN-BARR VIRUS}

Epstein-Barr virus (EBV) is a gamma-herpesvirus with a $172-\mathrm{kb}$ DNA genome, which infects more than $90 \%$ of world population. EBV is a successful virus that utilizes normal B cell biology to infect, persist, and replicate in B cells (Thorley-Lawson, 2001; ThorleyLawson and Gross, 2004). EBV predominantly infects resting B lymphocytes through complement receptor 2 (CD21) in vitro (for a review of EBV biology, Young and Rickinson, 2004). Primary infection of EBV may cause short term proliferation of B cells in human hosts. The infection is usually self-limited and controlled by the strongly elevated $\mathrm{T}$ cell immune response. If the infection occurs in adolescence or adulthood, up to 50\% T cells in the host can be specific to the virus, which may cause the clinical symptom of infectious mononucleosis (IM). EBV then persists latently in the host within long-life memory B cells.

During latency, up to eight EBV encoded proteins and several non-coding RNAs are expressed. These include two EBV encoded small RNAs (EBER1 and EBER2), nuclear antigens, and membrane proteins. EBV nuclear antigen 1 (EBNA-1) binds to the latent viral DNA replication origin and maintains the viral genome in the EBV positive cells after cell division (Yates et al., 1985). EBNA-2 interacts with a DNA binding protein $\mathrm{CBF} 1$ through mimicking Notch signaling pathway, blocks differentiation, and allows cell proliferation (Ling et al., 1994). Two EBV latent membrane proteins (LMPs) adopt the signaling pathways involved in B cell activation and differentiation to sustain the long-life of EBV positive cells. Both LMP1 and LMP2A are multiple membrane-spanning proteins that function as constitutive active receptors independent of ligand binding (Gires et al., 1997). On one hand, the carboxyl terminus of LMP1 contains consensus tumor-necrosis-factor- receptor-associated factor (TRAF)-binding domains and interacts with multiple members, in a B cell activation molecule CD40 mimicking pattern that can activate STAT, JNK, and NF- $\kappa$ B pathways and lead to B cell survival and growth. On the other hand, LMP2A contains immunoreceptor tyrosine-based activation motifs (ITAMs) and associates with Lyn kinase, and LMP2A can then replace the survival signal provided by $\mathrm{B}$ cell receptor $\mathrm{BCR}$; Caldwell et al., 1998). Overall, the EBV-infected naïve B cell blasts proliferate in a way that resembles the antigen-activated blasts (Thorley-Lawson and Mann, 1985). However, different from terminally differentiated plasma cells that eventually undergo apoptosis, these EBV positive cells follow the path of B cell differentiation into memory B cells through migration into germinal center (GC; Thorley-Lawson, 2001). Four different types of latency programs were defined according to the expression profile of EBV latent genes. These memory cells do not express EBNA-1 or other latent proteins (referred as latency 0 ) under normal condition, but only express EBNA-1 when cells are dividing (Hochberg et al., 2004). In vitro, the EBV transformed lymphoblastoid cell line (LCL) with all eight viral latent proteins, EBV encoded small RNA (EBERs), and BamHI A transcripts can be continuously propagated. EBNA-1, EBNA-2, EBNA-3A, EBNA-LP, and LMP1 are required for this transformation process (Kutok and Wang, 2006). Because of the strong immunogenicity of EBV latent proteins, the LCL-like viral gene expression pattern (Type III latency), can only be observed in immunocompromised patients in vivo. Alternatively, EBV positive Burkitt's lymphoma (BL) cells express only EBNA-1 and EBV encoded RNAs (Type I latency), whereas cells with Type II latency expressing also LMP1 and LMP2 are observed in Hodgkin's disease (HD). 


\section{EBV AND ITS ASSOCIATED MALIGNANT DISEASES}

The above mentioned information indicates that after primary infection, a subset of EBV positive B cells survive and emerge into the memory compartment. These cells can go for limited expansion or extrafollicular proliferation and sustain in the host for a longtime. The virus can be reactivated periodically and infect new hosts through shedding in saliva. In addition to B cell, EBV also infects epithelial cells and maybe some T cells. EBV is also highly associated with several neoplastic diseases, such as endemic BL, T cell lymphoma, nasopharyngeal carcinoma (NPC), HD, and immunoblastic lymphomas in immunocompromised patients (Young and Rickinson, 2004). Viral genomes or subsets of viral gene products can be detected in the tumor tissues, and antibody levels are usually elevated in patients' sera. Though not fully illustrated, recent knowledge provides some hints to the EBV associated disease mechanisms.

The EBV associated BL (endemic BL in tropic Africa) demonstrates that the complex interaction of $\mathrm{EBV}$ with $\mathrm{B}$ cells predisposes the development of BL (for reviews, Brady et al., 2008; ThorleyLawson and Allday, 2008). Malaria infection in endemic region is considered as another co-factor for the development of BL. EBVnegative BL also occurred in other area, and myc-translocation to Ig locus is the most critical feature observed in both sporadic and endemic BLs. The translocation is highly dependent on the enzyme activity of AID (activation-induced cytidine deaminase; Muramatsu et al., 2000; Pasqualucci et al., 2008). AID is highly expressed in GC to provide the class switch and hypermutation of Ig variable region. After translocation, the activated myc on one hand can lead to cell growth and proliferation, but myc also leads to p53 and BIM dependent apoptosis. Effects of EBV proteins may prevent $\mathrm{B}$ cells from myc induced apoptosis, thus sustain the survival of these memory B cells. The malaria infection may represent a chronic antigenic stimulation with repeated opportunistic infections that can promote more EBV-infected cells to become memory cells. It is still not clear whether chronic B cell antigen stimulation can link to the prolonged or atypical expression of AID, and lead to the higher frequency of myc-translocation (Thorley-Lawson and Allday, 2008).

Hodgkin's lymphoma (HL) is characterized by the presence of a minority of malignant Hodgkin/Reed Sternberg (HRS) cells among a background of non-neoplastic B and $\mathrm{T}$ cells (For a review, Kapatai and Murray, 2007). A complex cytokine cross-talk among HRS cells and neighboring cells may provide proliferative and anti-apoptotic signals for tumor cell growth. According to the WHO classification, HL can be divided into two major types: classical and nodular lymphocyte predominant HL (Harris et al., 1999). EBV is associated with only subsets of classical HL. Nevertheless, EBV positive and negative HLs share similar morphological patterns, while different signaling pathways are involved in the pathogenesis. Most EBV positive HLs belong to the mixed cellularity subtype in classical HL. EBV positive HRS cells display type II latency gene expression profile with EBERs, EBNA-1, LMP1 and LMP2, and BamHI A rightward transcripts (BARTs). The lack of expression of functional surface immunoglobulin is the hall mark of classical HL. But because of the clonally rearranged immunoglobulin genes identified in HRS cell, the B cell origin of HL is confirmed (Kuppers et al.,
1994). Under normal situation, antigen-activated B cells go to GC for somatic hypermutation to generate high affinity BCRs, and those cells with unfavorable mutations will be eliminated through Fas-mediated apoptosis. The current scenario for HL is that the expression of EBV genes may prevent the apoptosis of cells with certain mutations and promote them into HRS cells (Kapatai and Murray, 2007).

\section{EBV AND NKT CELL DEVELOPMENT}

It has been a longtime question why EBV only causes these malignant diseases within a small portion of infected population. Recent studies further indicated that EBV infection may regulate $\mathrm{CD} 8^{+}$ NKT development in the host; and the failure of $\mathrm{CD}^{+} \mathrm{NKT}$ conversion may contribute to disease development in some cases (He et al., 2010). NKT cells are unconventional T cells that are CD1d-restricted, lipid antigen-reactive, immune regulatory T lymphocytes. NKT cells can produce a broad range of cytokines upon antigen stimulation within minutes to hours. Different subsets of NKT are involved in regulating cell-mediated immunity to various infectious organisms, cancer, allergy, and autoimmune diseases. It is still unclear why such a small subset of $\mathrm{T}$ cells [ $\mathrm{T}$ cell receptor (TCR)- $\alpha$ V $\alpha 14-J \alpha 18$ ] can have such broad influence. Most notably, NKT cell numbers vary substantially between individuals (from undetectable to over $3 \%$ of blood lymphocytes). This may be either the result of differences in intrathymic development, migration efficiency or the maintenance, or expansion in peripheral blood (for a review, Godfrey et al., 2010). Generally speaking, type I NKT cells can mediate strong antitumor responses on $\alpha$-GalCer-induced IFN- $\gamma$ and drive T cells into Th1-bias. Type II NKT cells suppress tumor immunity through IL-13 secretion (Berzofsky and Terabe, 2008). It is therefore attractive to study the NKT population variation in different diseases and ask how the NKT population could be manipulated.

A recent study revealed that the frequencies of regulatory $\mathrm{CD} 8^{+}$ NKT, but not $\mathrm{CD}^{+}$NKT cells, in EBV associated HL and NPC patients are much lower than that in healthy EBV carriers (Yuling et al., 2009). In the human-thymus-sever combined immunodeficient (hu-thy-SCID) chimera mouse model, the authors further demonstrated that EBV promotes the generation of IFN- $\gamma$-biased EBV specific $\mathrm{CD}^{+} \mathrm{NKT}$ cells, which can suppress tumorigenesis by EBV associated malignancies in vivo (Xiao et al., 2009; Yuling et al., 2009). To further illustrate the interplay between EBV and NKT development, the same group demonstrated that the average frequency of total and $\mathrm{CD}^{+} \mathrm{NKT}$ cells in peripheral blood mononuclear cells (PBMCs) of healthy EBV latent individuals is significantly higher than that in patients with acute EBV IM or $\mathrm{HL}$, and in EBV-negative normal control subjects. EBV challenge induced a population of NKT precursors develops and differentiates into mature $\mathrm{CD} 8^{+} \mathrm{NKT}$ cells in the thymus and liver of humanthymus/liver-SCID chimera mouse model. These $\mathrm{CD}^{+} \mathrm{NKT}$ cells produce more perforin and are $\mathrm{CD} 8 \alpha \alpha$ positive, similar to that detected on $\mathrm{CD}^{+}$NKT cells in PBMCs from healthy latent EBVinfected subjects and IM patients at 1 year post-onset. The authors proposed that thymic EBV-infected dendritic cells and IL-7 may regulate the $\mathrm{CD} 8^{+} \mathrm{NKT}$ development process. It clearly demonstrated that EBV can induce differential CD4 versus CD8 lineage commitment, in addition to known classical endogenous elements 
(He et al., 2010). Thus the distinctive subsets of $\mathrm{CD} 4^{+}$and $\mathrm{CD} 8^{+}$ NKT cells in EBV-infected individuals may differentially modify the pathogenesis of EBV. Alternatively, it may be possible that the biased NKT will affect the specific individual's response to later infections or malignant diseases.

\section{EBV AND AUTOIMMUNE DISEASES}

Keeping the above observation in mind, we may check possible mechanisms involved in EBV associated autoimmune diseases, such as systemic lupus erythematosus (SLE) and rheumatoid arthritis (RA; for a review, Toussirot and Roudier, 2008). Both SLE and RA are complex disorders with a genetic background, as suggested by familial cases. Patients of both diseases have higher titers of anti-EBV antibodies, impaired $\mathrm{T}$ cell responses to EBV antigens and higher viral load in PBMCs (James et al., 2001; Poole et al., 2006). EBV DNA or RNA was detected in the target organs of RA. Molecular mimicry between EBV and lupus autoantigens was reported. Antibodies against cross-reactive epitopes containing glycine-alanine rich sequences and cellular nuclear antigens were identified (Vaughan et al., 1995). A proposed disease mechanism is the interplay between genetic predisposition and EBV infection leads to the development of cross reacting autoantibodies, and later the non-cross-reactive antibodies against autoepitopes (Toussirot and Roudier, 2008).

In addition to SLE and RA, multiple sclerosis (MS) is another EBV associated chronic inflammatory disease with repetitive damages of myelin sheet in the central nervous system (CNS). MS is a disease that also has been linked to genetic and environmental factors (for a review, Compston and Coles, 2008). Based on the geographic distribution, MS was proposed to be a rare disease that caused by a common infection. The most promising data suggesting EBV as the viral factor is that the risk of MS is 20 times higher among people who have contracted IM, as compared with seronegative individuals (Ascherio and Munger, 2007). In a large scale follow-up study for MS development in US military, the risk of MS increased with elevated levels of serum antibodies to EBNA complex and less strongly to VCA IgG. Some of the serum antibody levels were elevated even 5 years before disease development (Levin et al., 2005; Compston and Coles, 2008). Detection of EBV specific antibodies in MS patients also showed a highly correlation in other studies. However because EBV is highly prevalent in healthy population, it has been a long-term debate on the role of EBV as the etiology agent of MS. The detection of EBV DNA or viral proteins by different groups then gave controversial results, which may be due to different samples included or the sensitivities of assays employed (Lunemann and Munz, 2009).

Recent detection of EBV markers in the postmortem brain tissue from patients with secondary progressive phase of MS thus provide a clue for the pathogenic contribution of EBV to MS patients. The breakthrough study by Serafini et al. (2007) demonstrated that almost $100 \%$ of the early onset MS cases with the secondary progressive phase contain dysregulated EBV infected plasma cells in MS brains. LMPs were regularly detected in MS brains, whereas viral lytic proteins were only detected in ectopic $\mathrm{B}$ cell follicles and acute lesions. Notably, activated $\mathrm{CD} 8^{+} \mathrm{T}$ cells with membrane associated perforin staining were observed at sites of major accumulation of EBV-infected cells, suggesting
T cell cytotoxicity toward EBV positive plasma cells (Serafini et al., 2007). Further study of the intrathecal B cells indicated that these cells are $\mathrm{CD} 27^{+}$antigen-experienced cells and with the co-expression of LMP1 and 2A. Moreover, LMP2A positive cells also express B cell-activating factor of the tumor-necrosis factor family (BAFF), suggesting these cells are responsible for persistent intrathecal synthesis of oligoclonal antibodies. The EBV positive cell pattern is very similar to the ectopic follicles observed in the tonsil of healthy EBV carriers. The disease model of MS was proposed by the authors that through the proliferative, anti-apoptotic, and B cell activation signals provided by EBV latent proteins, EBV may promote the intracerebral expansion and maturation of B cells and form ectopic lymphoid follicles. It also explains that the oligoclonal IgG in the CSF and brain tissue is synthesized persistently by EBV positive B cells (Serafini et al., 2010). There are still remaining questions. Why are the EBV positive B cells homing to brain? Are these EBV positive cells the cause or the consequence of MS?

Other possibilities for the contribution of EBV in MS are also proposed by other groups. The idea is that virus positive cells may be few, but these cells may induce enough pro-inflammatory cytokine production and lead to the development of disease. For example, EBV was demonstrated to infect primary human brain microvessel endothelial cells in vitro (Casiraghi et al., 2011). The infection also leads to increased production of several pro-inflammatory mediators, such as CCL-5 and ICAM-1, which may promote adhesion of leukocytes to the endothelium. It is likely that the entry of autoreactive $\mathrm{T}$ cells may follow a breach of the blood-brain barrier (BBB) and lead to CNS lesions in MS. It explains why viral and myelin-specific lymphocytes and macrophages infiltrate into MS brains, and interferon-beta treatment may prevent MS relapse through blocking virus replication and subsequent production of cytokines (Casiraghi et al., 2011). Alternatively, another recent study focused on the development of $\mathrm{CD}^{+}$cytotoxic $\mathrm{T}$ cells observed in MS patients (Ji et al., 2010). The authors used a major histocompatibility complex (MHC) class I-restricted TCR transgenic model that generates $\mathrm{CD}^{+}$myelin basic protein (MBP) specific $\mathrm{T}$ cells to study the break of $\mathrm{CD} 8^{+} \mathrm{T}$ cell tolerance and induction of CNS autoimmunity. The results indicate that viral infection triggers the activation of $\mathrm{CD}^{+} \mathrm{T}$ cells bearing dual TCRs in the experimental animals, suggesting these $\mathrm{T}$ cells may contribute to CNS autoimmune diseases (Ji et al., 2010; Ransohoff, 2010). Indeed, dual-receptor TCRs are present in humans and proposed to enhance antiviral defense. The second receptor could be against myelin in a small subset of individuals, which may be associated with specific MHC II subtypes. It is possible that reinfection or reactivation of the specific virus may activate the $\mathrm{T}$ cell antiviral memory and also the anti-myelin activity (Ransohoff, 2010). The question then is why infection of EBV, but not other viruses, links to the development of MS.

We may then think again the stimulatory effect of EBV on NKT development, regarding the viral modulation on immune system (He et al., 2010). It was previously known that the infection of HIV or HTLV-1 may result in a decrease in NKT cells (Lin et al., 2005; Azakami et al., 2009). It thus indicates that various viral infections may have different impacts on the development of host immune response, and EBV may be a very unique virus. From an evolution 
point of view, it is plausible that EBV may serve certain roles to help immune system development in most population. The EBV associated diseases may be rare events resulting from host genetic variations, which let the host immune system be modified by EBV in a bias manner. The disease statuses may develop upon the stimulation of combinatory environmental factors such as other infectious agents, which may activate EBV replication at the same time. In other words, these EBV associated diseases may be due to the differential immune modulation ability of EBV in people with different genetic background, rather than a direct pathogenic effect of EBV gene products. Taken together, multi-factorial study

\section{REFERENCES}

Ascherio, A., and Munger, K. L. (2007). Environmental risk factors for multiple sclerosis. Part I: the role of infection. Ann. Neurol. 61, 288-299.

Azakami, K., Sato, T., Araya, N., Utsunomiya, A., Kubota, R., Suzuki, K., Hasegawa, D., Izumi, T., Fujita, H., Aratani, S., Fujii, R., Yagishita, N., Kamijuku, H., Kanekura, T., Seino, K., Nishioka, K., Nakajima, T., and Yamano, Y. (2009). Severe loss of invariant NKT cells exhibiting antiHTLV-1 activity in patients with HTLV-1-associated disorders. Blood 114, 3208-3215.

Berzofsky, J. A., and Terabe, M. (2008). NKT cells in tumor immunity: opposing subsets define a new immunoregulatory axis. J. Immunol. 180, 3627-3635.

Brady, G., Macarthur, G. J., and Farrell, P. J. (2008). Epstein-Barr virus and Burkitt lymphoma. Postgrad. Med. J. 84, 372-377.

Caldwell, R. G., Wilson, J. B., Anderson, S. J., and Longnecker, R. (1998). Epstein-Barr virus LMP2A drives B cell development and survival in the absence of normal B cell receptor signals. Immunity 9, 405-411.

Casiraghi, C., Dorovini-Zis, K., and Horwitz, M. S. (2011). Epstein-Barr virus infection of human brain microvessel endothelial cells: a novel role in multiple sclerosis. J. Neuroimmunol. 230, 173-177.

Compston, A., and Coles, A. (2008). Multiple sclerosis. Lancet 372, 1502-1517.

Gires, O., Zimber-Strobl, U., Gonnella, R., Ueffing, M., Marschall, G., Zeidler, R., Pich, D., and Hammerschmidt, W. (1997). Latent membrane protein 1 of Epstein-Barr virus mimics a constitutively active receptor molecule. $E M B O$ J. 16, 6131-6140.

Godfrey, D. I., Stankovic, S., and Baxter,A. G. (2010). Raising the NKT cell family. Nat. Immunol. 11, 197-206.

Harris, N. L., Jaffe, E. S., Diebold, J., Flandrin, G., Muller-Hermelink, H.
K., Vardiman, J., Lister, T. A., and Bloomfield, C. D. (1999). The world health organization classification of neoplastic diseases of the hematopoietic and lymphoid tissues. Report of the clinical advisory committee meeting, airlie house, Virginia, November, 1997. Ann. Oncol. 10, 1419-1432.

He, Y., Xiao, R., Ji, X., Li, L., Chen, L., Xiong, J., Xiao, W., Wang, Y., Zhang, L., Zhou, R., Tan, X., Bi, Y., Jiang, Y. P., Jin, Y., and Tan, J. (2010). EBV promotes human CD8 NKT cell development. PLoS Pathog. 6, e1000915. doi: 10.1371/journal.ppat.1000915

Hochberg, D., Middeldorp, J.M., Catalina, M., Sullivan, J. L., Luzuriaga, K., and Thorley-Lawson, D. A. (2004). Demonstration of the Burkitt's lymphoma Epstein-Barr virus phenotype in dividing latently infected memory cells in vivo. Proc. Natl. Acad. Sci. U.S.A. 101, 239-244.

James, J. A., Neas, B. R., Moser, K. L., Hall, T., Bruner, G. R., Sestak, A. L., and Harley, J. B. (2001). Systemic lupus erythematosus in adults is associated with previous Epstein-Barr virus exposure. Arthritis Rheum. 44, 1122-1126.

Ji, Q., Perchellet, A., and Goverman, J. M. (2010). Viral infection triggers central nervous system autoimmunity via activation of CD8+ T cells expressing dual TCRs. Nat. Immunol. 11, 628-634.

Kapatai, G., and Murray, P. (2007). Contribution of the Epstein Barr virus to the molecular pathogenesis of Hodgkin lymphoma. J. Clin. Pathol. 60, 1342-1349.

Kuppers, R., Rajewsky, K., Zhao, M., Simons, G., Laumann, R., Fischer, R., and Hansmann, M.L. (1994). Hodgkin disease: Hodgkin and Reed-Sternberg cells picked from histological sections show clonal immunoglobulin gene rearrangements and appear to be derived from $B$ cells at various stages of development. Proc. Natl. Acad. Sci. U.S.A. 91, 10962-10966.

Kutok, J.L., and Wang, F. (2006).Spectrum of Epstein-Barr virus-associated diseases. Annu. Rev. Pathol. 1, 375-404.

designs including genetic factors, individual variations of subsets of regulatory immune cells, and environmental factors such as different infections are required for understanding the pathogenic mechanism of EBV associated diseases in the future.

\section{ACKNOWLEDGMENTS}

The author would like to thank the Editor and reviewers for their critical reading and suggestions. The author's research is supported by National Science Council (NSC98-2320B002-54 MY3), National Health Research Institutes (NHRI-EX99-9928BI) and National Taiwan University (99R71423), Taiwan.

Levin, L. I., Munger, K. L., Rubertone, M. V., Peck, C. A., Lennette, E. T. Spiegelman, D., and Ascherio, A. (2005). Temporal relationship between elevation of Epstein-Barr virus antibody titers and initial onset of neurological symptoms in multiple sclerosis. JAMA 293, 2496-2500.

Lin, Y., Roberts, T. J., Wang, C. R., Cho, S., and Brutkiewicz, R. R. (2005). Longterm loss of canonical NKT cells following an acute virus infection. Eur. J. Immunol. 35, 879-889.

Ling, P. D., Hsieh, J. J., Ruf, I. K., Rawlins, D. R., and Hayward, S. D. (1994) EBNA-2 upregulation of Epstein-Barr virus latency promoters and the cellular CD23 promoter utilizes a common targeting intermediate, CBF1. J. Virol. $68,5375-5383$.

Lunemann, J. D., and Munz, C. (2009) EBV in MS: guilty by association? Trends Immunol. 30, 243-248.

Muramatsu, M., Kinoshita, K., Fagarasan, S., Yamada, S., Shinkai, Y., and Honjo T. (2000). Class switch recombination and hypermutation require activationinduced cytidine deaminase (AID), a potential RNA editing enzyme. Cell 102, 553-563.

Pasqualucci, L., Bhagat, G., Jankovic, M., Compagno, M., Smith, P., Muramatsu, M., Honjo, T., Morse, H. C. III, Nussenzweig, M. C., and Dalla-Favera, R. (2008).AID is required for germinal center-derived lymphomagenesis. Nat. Genet. 40, 108-112.

Poole, B. D., Scofield, R. H., Harley, J. B., and James, J. A. (2006). EpsteinBarr virus and molecular mimicry in systemic lupus erythematosus. Autoimmunity 39, 63-70.

Ransohoff, R. M. (2010). Turning over the chance card on MS susceptibility. Nat. Immunol. 11, 570-572.

Serafini, B., Rosicarelli, B., Franciotta, D., Magliozzi, R., Reynolds, R., Cinque, P., Andreoni, L., Trivedi, P., Salvetti, M., Faggioni, A., and Aloisi, F. (2007). Dysregulated Epstein-Barr virus infection in the multiple sclerosis brain. $J$. Exp. Med. 204, 2899-2912.
Serafini, B., Severa, M., ColumbaCabezas, S., Rosicarelli, B., Veroni, C., Chiappetta,G.,Magliozzi, R., Reynolds, R., Coccia, E. M., and Aloisi, F. (2010). Epstein-Barr virus latent infection and BAFF expression in B cells in the multiple sclerosis brain: implications for viral persistence and intrathecal B-cell activation. J. Neuropathol. Exp. Neurol. 69, 677-693.

Thorley-Lawson, D. A. (2001). EpsteinBarr virus: exploiting the immune system. Nat. Rev. Immunol. 1, 75-82.

Thorley-Lawson, D. A., and Allday, M. J. (2008). The curious case of the tumour virus: 50 years of Burkitt's lymphoma. Nat. Rev. Microbiol. 6, 913-924.

Thorley-Lawson, D. A., and Gross, A. (2004). Persistence of the EpsteinBarr virus and the origins of associated lymphomas. N. Engl. J. Med. 350, 1328-1337.

Thorley-Lawson, D. A., and Mann, K. P. (1985). Early events in Epstein-Barr virus infection provide a model for B cell activation. J. Exp. Med. 162, 45-59.

Toussirot, E., and Roudier, J. (2008). Epstein-Barr virus in autoimmune diseases. Best Pract. Res. Clin. Rheumatol. 22, 883-896.

Vaughan, J. H., Valbracht, J. R., Nguyen, M. D., Handley, H. H., Smith, R. S. Patrick, K., and Rhodes, G. H. (1995). Epstein-Barr virus-induced autoimmune responses. I. Immunoglobulin Mautoantibodies to proteins mimicking and not mimicking Epstein-Barr virus nuclear antigen-1. J. Clin. Invest. 95, 1306-1315.

Xiao, W., Li, L., Zhou, R., Xiao, R., Wang, Y., Ji, X., Wu, M., Wang, L., Huang, W., Zheng, X., Tan, X., Chen, L., Xiong, T., Xiong, J., Jin, Y., Tan, J., and He, Y. (2009). EBV-induced human CD8(+) NKT cells synergise CD4(+) NKT cells suppressing EBV-associated tumours upon induction of Th1-bias. Cell. Mol. Immunol. 6, 367-379.

Yates, J. L., Warren, N., and Sugden, B. (1985). Stable replication of plasmids 
derived from Epstein-Barr virus in various mammalian cells. Nature $313,812-815$.

Young, L. S., and Rickinson, A. B. (2004). Epstein-Barr virus: 40 years on. Nat. Rev. Cancer 4, 757-768.

Yuling, H., Ruijing, X., Li, L., Xiang, J., Rui, Z., Yujuan, W., Lijun, Z., Chunxian, D., Xinti, T., Wei, X., Lang, C., Yanping, J., Tao, X., Mengjun, W., Jie, X.,
Youxin, J., and Jinquan, T. (2009). EBV-induced human CD8+ NKT cells suppress tumorigenesis by EBVassociated malignancies. Cancer Res. 69, 7935-7944.

Conflict of Interest Statement: The author declares that the research was conducted in the absence of any commercial or financial relationships that could be construed as a potential conflict of interest.

Received: 29 September 2010; accepted: 11 January 2011; published online: 26January 2011.

Citation: Chen M-R (2011) Epstein-Barr virus, the immune system, and associated diseases. Front. Microbio. 2:5. doi: 10.3389/ fmicb.2011.00005
This article was submitted to Frontier in Virology, a specialty of Frontiers in Microbiology.

Copyright $(02011$ Chen. This is an openaccess article subject to an exclusive license agreement between the authors and Frontiers Media SA, which permits unrestricted use, distribution, and reproduction in any medium, provided the original authors and source are credited. 